\title{
MicroRNA-152 suppresses cell proliferation and tumor growth of bladder cancer by targeting KLF5 and MKK7
}

\author{
Yu Hui ${ }^{a}$, Yuhua Huang ${ }^{a}$, Xiang Ding ${ }^{a}$, Liangliang Wang ${ }^{a, *}$ \\ ${ }^{a}$ Department of Urology, The First Affiliated Hospital of Soochow University, Suzhou, Jiangsu, China.
}

\begin{abstract}
Background: In bladder cancer, miR-152 has been reported to be downregulated and accompanied by high DNA methylation. However, the molecular mechanisms underlying miR-152 in bladder cancer tumorigenesis remain unknown.

Methods: The expressions of miR-152 in human bladder cancer cell lines (J82, 5637, and T24) and a human bladder epithelial cell line (SV-HUC-1) were compared. The expression of miR-152 was observed after adding DNA methylation inhibitor 5-Aza-dC or after knocking down the major DNA methyltransferase DNMT1. The bladder cancer cell viability was determined using an MTT assay, and the colony formation rate was determined using a colony formation assay. The effects of miR-152 overexpression on cell proliferation and tumor growth were observed in vitro and in vivo. The interactions among miR-152, KLF5, and MKK7 were determined using a luciferase reporter assay.

Results: MiR-152 was downregulated in bladder cancer cell lines in comparison with normal ones. The expression of miR-152 was significantly promoted after adding 5-Aza-dC or after knocking down DNMT1. The overexpression of miR-152 suppressed cell proliferation and tumor growth both in vitro and in vivo. MiR-152 inhibited KLF5 and MKK7 expression by binding with 3' untranslated regions of their mRNAs. Meanwhile, the overexpression of KLF5 induced MKK7 expression by increasing the transcriptional activity of MKK7. The simultaneous overexpression of miR-152 and KLF5 reversed the inhibition of cell proliferation caused by miR152 overexpression alone.

Conclusion: Our findings suggest that miR-152 acts as a tumor suppressor in bladder cancer, and the overexpression of miR-152 may be a potential strategy for treating bladder cancer.
\end{abstract}

Keywords: Cell proliferation, bladder cancer, DNA methylation, miR-152, transcription factor

\section{Introduction}

Bladder cancer is one of the most common cancers in the human urinary system, and most bladder cancers (70-80\%) are non-muscle invasive, which is associated with a good prognosis [1]. However, about $10-15 \%$ of bladder cancers will recur, progress to a muscle invasive state, and even metastasize to multiple organs [2]. Therefore, it is vital to understand the mechanisms underlying bladder tumor growth and to explore new targets to treat bladder cancer. Kruppel-like factor 5 (KLF5), which is also known as

\footnotetext{
* Corresponding author: Liangliang Wang

Mailing address: Department of Urology, The First Affiliated Hospital of Soochow University, No.188 Shizi Rd, Gusu District, Suzhou 215006, Jiangsu, People's Republic of China. Email: w11234@163.com
}

Received: 18 December 2019 / Accepted: 20 December 2019
IKLF or BTEB2, belongs to the Kruppel-like transcription factor family [3]. As reported, KLF5 is a critical regulator in carcinogenesis, including in bladder cancer. For example, Chen et al. [4] demonstrated that KLF5 promotes bladder cancer cell proliferation by inducing G1 to $\mathrm{S}$ phase transition. Similarly, Du et al. [5] suggested that KLF5 promotes bladder cancer cell migration and lamellipodia formation by inducing the transcription of FYN. These studies reveal the oncogenic function of KLF5 in bladder cancer, whereas the molecular mechanisms of KLF5 in bladder cancer remain largely unknown. The mitogen-activated protein kinase (MAPK) pathway is a major cell-mediated cascade that regulates processes such as cell death, differentiation, growth, and stress response $[6,7]$. The activation of the c-Jun N-terminal kinase (JNK) cascade is involved in the MAPK signaling process [8]. As an important component of the JNK cascade, mitogenactivated protein kinase kinase 7 (MKK7) has been reported to act as an oncogene in several types of cancers, such as lung cancer [9] and colorectal cancer [10]. Nota- 
bly, a previous study has revealed that the transcriptional factor KLF5 could positively regulate MKK7 expression by binding with MKK7 promoter regions [11]. However, whether KLF5 interacts with MKK7 in regulating bladder cancer tumorigenesis remains unknown.

MicroRNAs (miRNAs) are a subset of non-coding RNAs (ncRNAs) consisting of about 20 nucleotides [12]. It is well known that miRNAs do not encode proteins and that they regulate gene expression at a posttranscriptional level. By binding with the 3' untranslated region (3'UTR) of a target mRNA, miRNAs promote the degradation of bound mRNA, thus suppressing gene expression [13]. MiR-152 is dysregulated in several cancers and acts as a tumor suppressor in several types of cancer, including breast cancer [14] and prostate cancer [15]. In bladder cancer, miR-152 was downregulated and accompanied by high DNA methylation [16], and serum miR-152 levels could provide information about the recurrence risk of non-muscle-invasive bladder cancer [17]. However, the regulatory pattern of miR-152 in bladder cancer has not been identified. Interestingly, we found that there were binding sites between miR-152 and KLF5, as well as between miR-152 and MKK7, by using an online prediction database. Herein, this study aimed to investigate whether miR-152 regulates bladder cancer cell proliferation and tumor growth by modulating KLF5 and MKK7.

\section{Materials and methods}

\section{Cell lines and cell culture}

Human bladder cancer cell lines (J82, 5637, and T24) and a human bladder epithelial cell line (SV-HUC-1) were bought from the American Type Culture Collection (ATCC). They were cultured in minimum Eagle's medium (MEM; Gibco, Waltham, MA, USA) supplemented with $10 \%$ fetal bovine serum (FBS; Thermo Fisher Scientific, Waltham, MA, USA).

\section{Quantitative real-time PCR}

The expressions of miR-152, MKK7 mRNA, and MKK7 mRNA in cells were detected using quantitative real-time PCR (qRT-PCR). Total RNAs were extracted from the tumor tissues and cells using Trizol reagent (Invitrogen, Waltham, MA, US). The TaqMan Reverse Transcription Kit (Applied Biosystems, Foster City, CA, US) was used to reversely transcribe RNAs into cDNAs. The qRT-PCR was conducted using the SYBR Premix Ex TaqTM II Kit (Takara, Dalian, Liaoning, China) on an ABI 7500 RealTime PCR system (Applied Biosystems, Waltham, MA, US). U6 was used as the internal control for miR-152, and GAPDH was used as the internal control for mRNAs. The relative expressions were calculated using the $2^{-\Delta \Delta \mathrm{Ct}}$ method.

\section{Cell transfection}

The small interfering RNA (siRNA) against DNMT1 (siDNMT1) and its control siRNA (si-NC), the miR-152 mimic and its negative control mimic (pre-NC), the miR152 inhibitor and its negative control (NC), the KLF5 overexpressing plasmid (pcDNA-KLF5) and its control plasmid (pcDNA), and the siRNA against KLF5 (si-KLF5) and its negative control siRNA (si-NC) were obtained from RiboBio Technology (Guangzhou, Guangdong, China). Cell transfection was performed using Lipofectamine 2000 reagents (Thermo Fisher Scientific, Waltham, MA, USA) according to the manufacturer's instructions.

\section{Cell proliferation assays}

Cell proliferation was detected using 3-(4, 5-dimethylthiazol-2-yl)-2, 5-diphenyltetrazolium bromide (MTT) and colony formation assays. After the transfection, cell viability was determined using an MTT assay (SigmaAldrich, St. Louis, MO, US) according to the manufacturer's protocol. For the colony formation assay, cells were seeded in 6-well plates at 1,000 cells per well. After 2 weeks, colonies were fixed with $4 \%$ paraformaldehyde and stained with crystal violet for $10 \mathrm{~min}$. Colonies consisting of more than 50 cells were counted.

\section{In vivo experiment}

The miR-152 overexpressing lentivirus (LV-miR-152) and the control lentivirus (LV-pre-NC) were obtained from RiboBio Technology (Guangzhou, Guangdong, China). Four-week-old male Balb/c nude mice $(n=10)$ were bought from the Shanghai Lab Animal Research Center (Shanghai, China). After the transfection of LV-miR-152/ Lv-pre-NC, the 5637 cells were subcutaneously injected into the nude mice. Tumor volume was measured every 3 days using a Vernier caliper. After 24 days, the mice were sacrificed, and the expression of miR-152 was detected using qRT-PCR. This study was approved by the Institute Research Medical Ethics Committee of the First Affiliated Hospital of Soochow University.

\section{Western blot}

A western blot was performed as previously described [11]. The primary antibodies used in this study included antiDNMT1 (\#5032S, 1:1,000, Cell Signaling Technology, Danvers, MA, USA), anti-KLF5 (\#51586S, 1:1,000, Cell Signaling Technology, Danvers, MA, USA), anti-MKK7 (\#4172S, 1:1,000, Cell Signaling Technology, Danvers, MA, USA), and anti- $\beta$-actin (ab8226, 1:500, Abcam, Cambridge, UK).

\section{Luciferase reporter assay}

To verify the relationship between miR-152 and KLF5/ MKK7, the wild type (WT) or mutant (Mut) 3'-UTR fragments of the predicted sequences of KLF5/MKK7 were amplified by PCR and cloned into a pISo vector (Promega, Madison, WI, US). The 5637 and T24 cells $\left(2 \times 10^{4} /\right.$ well $)$ were transfected with miR-152 mimic or miR-152 inhibitor and WT-KLF5/MKK7-3'UTR-pISo or Mut-KLF5/ MKK7-3'UTR-pISo, together with the control vectors (pre-NC or NC). After $24 \mathrm{~h}$, the cells were collected and subjected to luciferase assays using the Luciferase Re- 

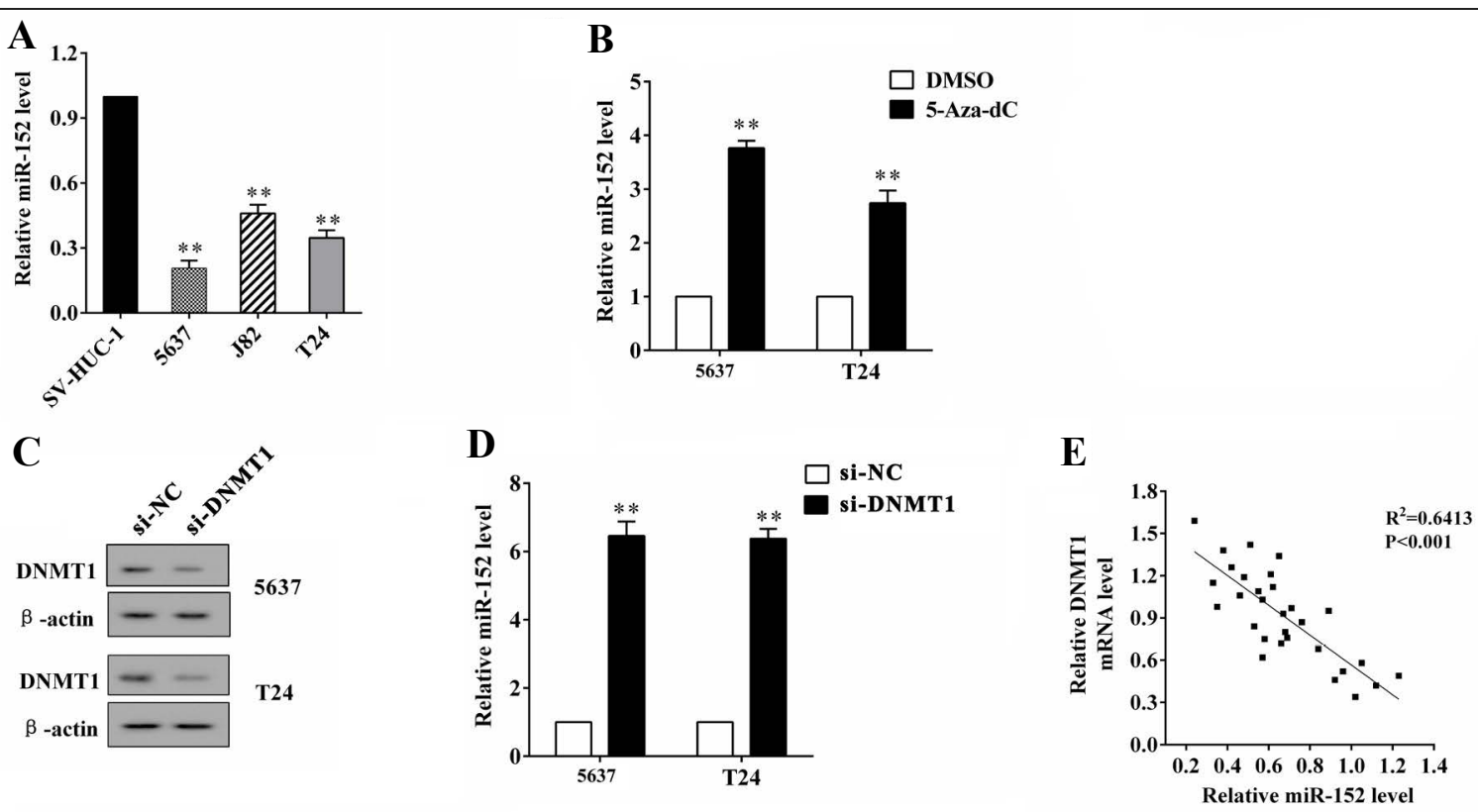

Figure 1. The MiR-152 expression in bladder cancer cells and the effect of DNMT1 on miR-152 expression. (A) The expression of miR-152 in bladder cancer cell lines $(5637, \mathrm{~J} 82$, and T24) and a normal bladder epithelial cell line (SV-HUC-1) was detected using qRT-PCR. (B) The DNA methylation inhibitor 5-Aza-dC or the control (DMSO) was added, and the expression of miR-152 in 5637 and T24 cells was detected using qRT-PCR. (C) The protein expression of DNMT1 in 5637 and T24 cells after the transfection of si-DNMT1 or the negative control (si-NC) was detected using a western blot analysis. (D) The expression of miR-152 in 5637 and T24 cells after the transfection of si-DNMT1 or the negative control (si-NC) was detected using qRT-PCR. (E) The correlation between DNMT1 mRNA and miR-152 was analyzed using a Pearson correlation analysis. ** $P<0.01$ vs. controls (SV-HUC-1, DMSO, and si-NC).

porter Assay System (Promega, Madison, WI, US).

To verify the effect of KLF5 on the promoter activity of MKK7, the promoter sequences of MKK7 (Lu-MKK7) or the control sequences (Lu-ctrl) were cloned into a pGL3 plasmid (Thermo Fisher Scientific, Waltham, MA, USA). The 293T cells were transfected with pcDNA-KLF5/ pcDNA and Lu-MKK7/Lu-ctrl for $48 \mathrm{~h}$. The Luciferase Reporter Assay System (Promega, Madison, WI, US) was used to detect the relative luciferase activity.

\section{Statistical analysis}

All data are presented as mean \pm standard deviation (SD). SPSS 18.0 and Graphpad Prism 5.0 were used for data analysis and imaging. Statistical analyses of the differences between the 2 groups were conducted using Stu-dent's $t$-test. Statistical analyses among multiple groups were conducted using the one-way analysis of variance (ANOVA) followed by the LSD post-hoc test. The cor-relation between miR-152 and DNMT1 mRNA was ana-lyzed using the Pearson correlation analysis. Differences were considered statistically significant if the $P$-value was less than 0.05 .

\section{Results}

MiR-152 is lowly expressed in bladder cancer cells and is negatively regulated by DNMT1

The expression of miR-152 was markedly lower in bladder cancer cell lines (5637, J82, and T24) than in a normal bladder epithelial cell line (SV-HUC-1) $(P<0.01$, Figure 1A). To observe the effects of DNA methylation on miR152 expression, the DNA methylation inhibitor 5-Aza-dC was added to suppress DNA methylation, and the miR152 expression was dramatically promoted in both 5637 and T24 cells $(P<0.01$, Figure 1B). After knocking down DNMT1 (Figure 1C), which is an important methyltransferase, the expression of miR-152 was markedly induced $(P<0.01$, Figure 1D). In addition, the expression of DNMT1 mRNA was negatively correlated with miR-152 expression $(P<0.001$, Figure $1 \mathrm{E})$.

Overexpression of miR-152 suppresses cell proliferation and tumor growth in vitro and in vivo

Given that miR-152 was downregulated in bladder cancer cells, we further investigated the effect of miR-152 overexpression on bladder cancer cell proliferation. The overexpression of miR-152 at concentrations of 25, 50, and $75 \mathrm{nM}$ suppressed cell viability after $48 \mathrm{~h}$ and $72 \mathrm{~h}$ of transfection in 5637 cells (all $P<0.01$, Figure 2A) and in T24 cells (all $P<0.01$, Figure $2 \mathrm{~B}$ ), respectively. Meanwhile, the overexpression of miR-152 reduced the colony formation rate in 5637 cells and T24 cells (both $P<0.01$, Figure $2 \mathrm{C}$ ). In the nude mouse xenograft model, the overexpression of miR-152 by the subcutaneous injection of 5637 cells carrying LV-miR-152 (Figure 2D) significantly inhibited tumor growth $(P<0.01$, Figure $2 \mathrm{E})$.

MiR-152 regulates KLF5 and MKK7 expressions by targeting the 3'UTR of their mRNAs 

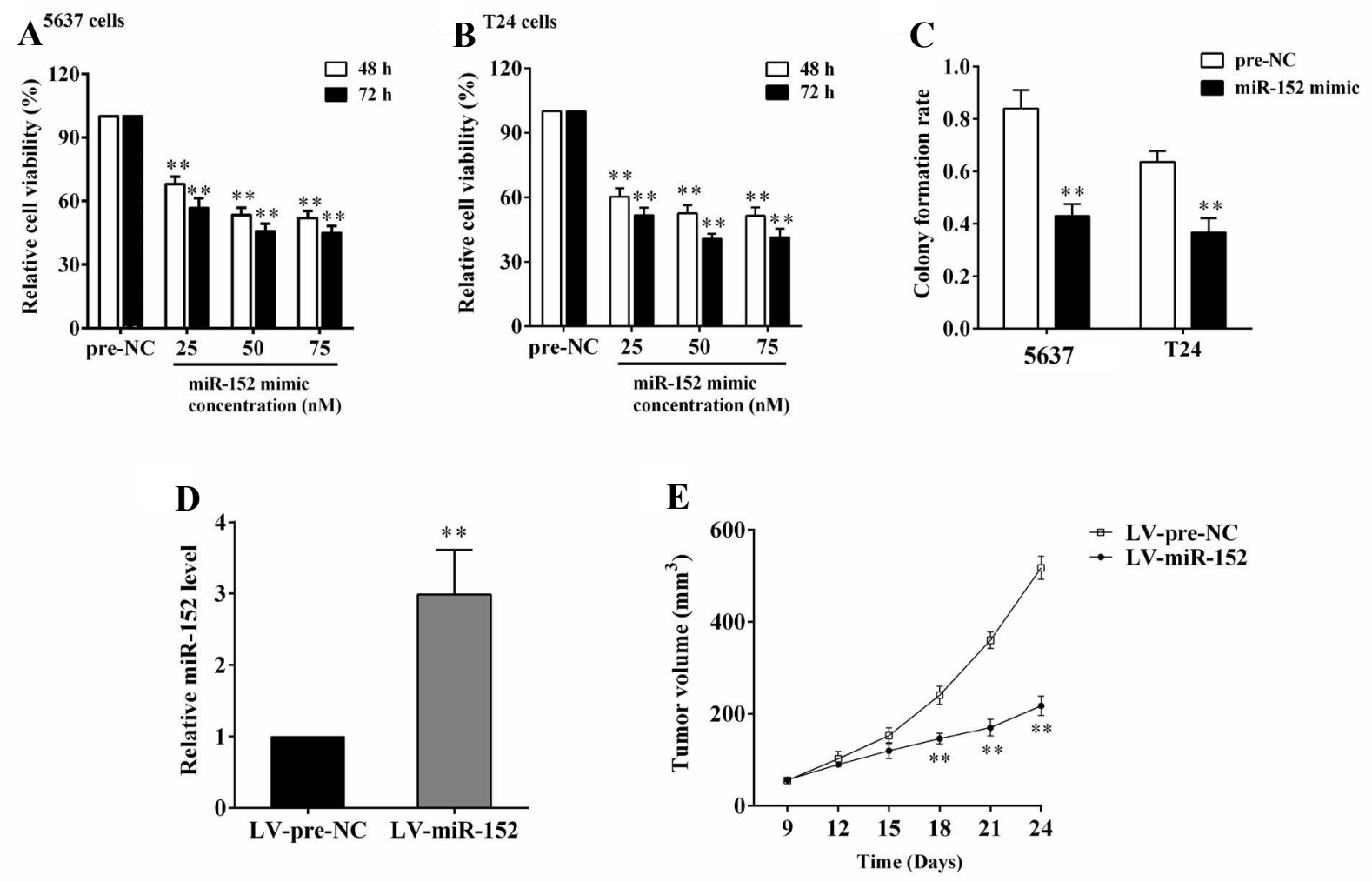

Figure 2. The effect of overexpressing miR-152 on cell proliferation and tumor growth in vitro and in vivo. The 5637 and T24 cells were transfected with miR-152 mimic at concentrations of 25,50 , and $75 \mathrm{nM}$. After $48 \mathrm{~h}$ or $72 \mathrm{~h}$, the cell viabilities of 5637 cells (A) and T24 cells (B) were detected, respectively, using an MTT assay. (C) The colony formation rate after miR-152 overexpression was detected using a colony formation assay. To induce the xenograft model, nude mice $(n=10)$ were subcutaneously injected with 5637 cells carrying LV-miR-152. The expression of miR-152 in tumor tissues (D) and tumor volume (E) were detected using qRT-PCR and a Vernier caliper, respectively. $* * P<0.01$ vs. controls (pre-NC or LV-preNC).

By searching the online database Targetscan (http:// www.targetscan.org), we found that there are binding sites between miR-152 and the 3'UTR of KLF5/MKK7 mRNAs (Figure 3A). When the predicted binding sites were mutated and cloned into a luciferase plasmid (Mut3'UTR KLF5), the relative luciferase activity was not significantly changed by either overexpressing or knocking down miR-152, whereas when the predicted binding sites were not mutated (WT-3'UTR KLF5), the relative luciferase activity was reduced by miR-152 overexpression and was enhanced by miR-152 knockdown $(P<0.01$, Figure $3 \mathrm{~B})$. Similar results were also observed when mutating or not mutating MKK7 3'UTR $(P<0.01$, Figure 3C). Meanwhile, miR-152 overexpression downregulated both mRNA and the protein levels of KLF5/MKK7, whereas miR-152 knockdown upregulated both the mRNA and protein levels of KLF5/MKK7 (Figure 3D, 3E). These data suggested that miR-152 suppressed KLF5 and MKK7 expressions via targeting the 3'UTR of their mRNAs. In addition, a previous study has revealed that the transcriptional factor KLF5 could positively regulate MKK7 expression by binding with MKK7 promoter regions [11]. In our study, the overexpression of KLF5 in 5637 and T24 cells significantly induced the mRNA expression of MKK7 $(P<0.01$, Figure 3F). The relative luciferase activity was also increased by KLF5 overexpression when the MKK7 promoter sequences were cloned into the lucif- erase plasmids $(P<0.01$, Figure $3 \mathrm{G})$.

\section{Overexpression of miR-152 suppresses cell prolifera- tion by modulating KLF5 and MKK7}

The 5637 and T24 cells were transfected with miR-152 inhibitor or co-transfected with siRNA against KLF5 (siKLF5). The transfection of miR-152 inhibitor markedly increased KLF5 and MKK7 expressions at the mRNA and protein levels, whereas the co-transfection negated these responses (Fiure 4A, B). These data indicate that miR152 regulated MKK7 expression by modulating KLF5. In addition, the overexpression of miR-152 suppressed cell viability and the colony formation rate, whereas the simultaneous overexpression of miR-152 and KLF5 negated these responses (Figure 4C, D), suggesting that miR-152 inhibited bladder cancer cell proliferation by downregulating KLF5.

\section{Discussion}

MiRNAs play a critical role in cell proliferation, differentiation, and apoptosis in cancers. In the current study, we found that miR-152 acted as a tumor suppressor in bladder cancer, and we also found that such effects were modulated by the targeting of KLF5 and MKK7. Our study provides a potential novel therapeutic target for bladder cancer. 


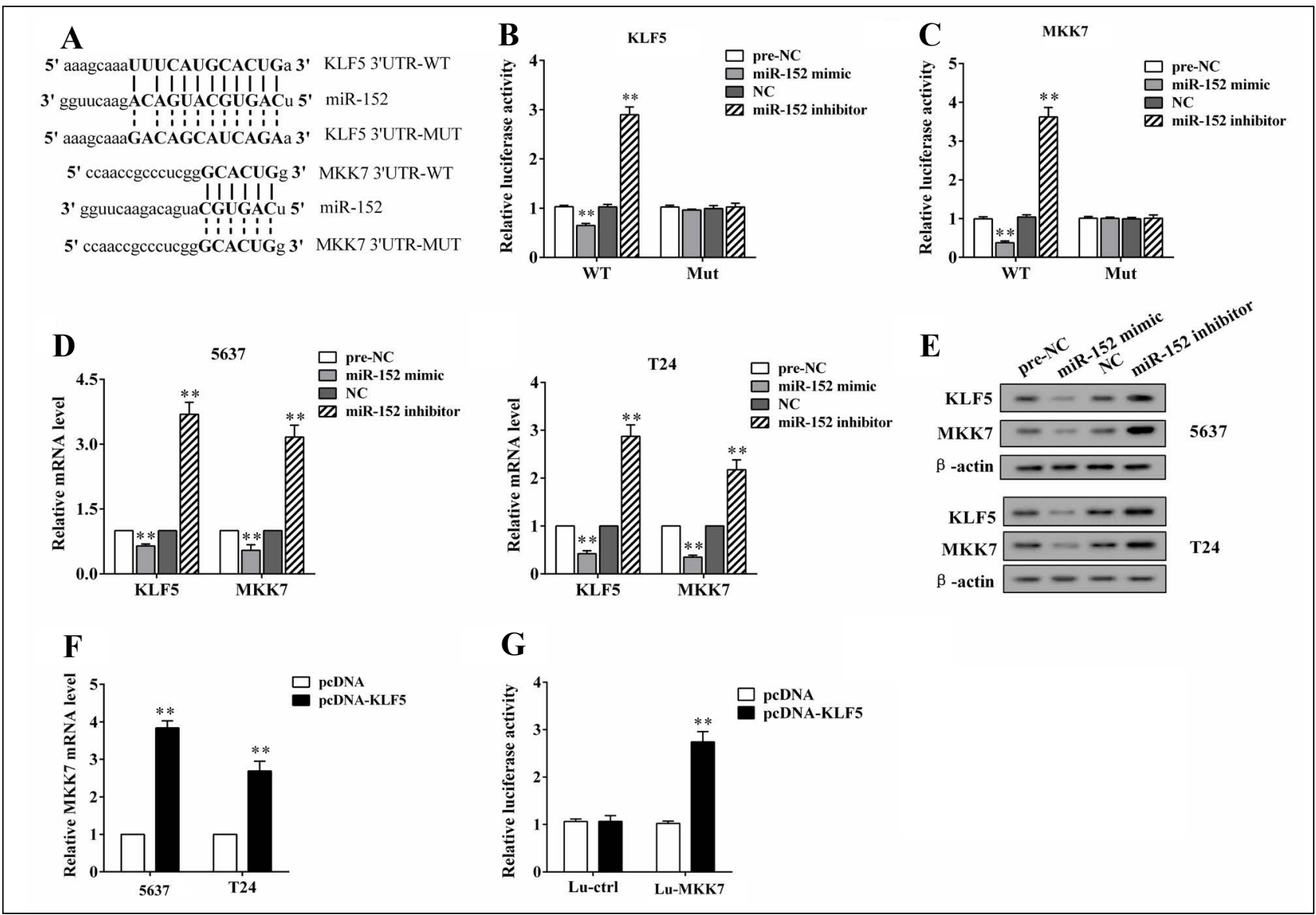

Figure 3. The relationship among miR-152, KLF5, and MKK7. (A) The predicted binding sites between miR-152 and the 3'UTR of KLF5/MKK7 mRNAs were found by searching the online database Targetscan. (B) The predicted binding sites were mutated (Mut-3'UTR KLF5) or not mutated (WT-3'UTR KLF5) and cloned into a luciferase plasmid, and the relative luciferase activity was detected using a luciferase reporter assay. (C) The predicted binding sites were mutated (Mut-3'UTR MKK7) or not mutated (WT-3'UTR MKK7) and cloned into a luciferase plasmid, and the relative luciferase activity was detected using a luciferase reporter assay. The 5637 and T24 cells were transfected with miR-152 mimic or inhibitor for $48 \mathrm{~h}$. The relative mRNA expression (D) and the protein expression (E) of KLF5 and MKK7 were detected using qRT-PCR and a western blot, respectively. (F) The 5637 and T24 cells were transfected with pcDNA-KLF5/pcDNA, and the mRNA expression of MKK7 was detected using qRT-PCR. (G) The MKK7 promoter sequences (Lu-MKK7) or the control sequences (Lu-ctrl) were cloned into a luciferase plasmid, and the relative luciferase activity was detected using a luciferase reporter assay. $* * P<0.01$ vs. controls (pre-NC, $\mathrm{NC}$, and pcDNA).

As reported, miRNAs have two effects on tumorigenesis, including oncogenic effects and anti-tumor effects. In bladder cancer, many miRNAs have been found to be involved in tumorigenesis. For instance, miR-9 [18, 19], miR-21 [20], and miR-19a [21] were upregulated in bladder cancer tumor tissues and acted as oncogenes, whereas miR-15b [22], miR-23b [23, 24], and miR-99a [25, 26] were downregulated in bladder cancer tumor tissues and acted as tumor suppressors. MiR-152 was reported to be involved in tumorigenesis and was downregulated in bladder cancer [16], and the serum miR-152 level was related to the recurrence risk of non-muscle-invasive bladder cancer [17]. Inspired by these studies, we investigated the molecular effects of miR-152 on bladder carcinogenesis and found that miR-152 was more lowly expressed in tumor cell lines than in controls. Meanwhile, the overexpression of miR-152 suppressed the proliferation of bladder cancer cells, suggesting the anti-tumor effects of miR152 overexpression. In addition, the overexpression of miR-152 markedly suppressed tumor growth in the mouse xenograft model. In summary, our findings indicate that the overexpression of miR-152 might be a therapeutic strategy for treating bladder cancer, although more evidence is still needed.

DNA methylation is an important DNA modification that affects gene expression. As an epigenetic regulation, the hypermethylation of DNA often results in a lower expression of the gene [27]. As a major DNA methyltransferase, DNMT1 [DNA (cytosine-5)-methyltransferase 1] transfers methyl groups into the $\mathrm{CpG}$ island on the DNA sequences, and it subsequently inhibits the transcription of genes [28]. Dysregulated DNA methylation is commonly seen in many types of cancers, and it is regarded as a main factor associated with tumorigenesis [29]. In bladder cancer, DNA hypermethylation was observed in miR-152 [16]. Consistent with these studies, we observed an elevation of miR-152 after the inhibition of DNA methylation by 5-Aza-dC. Meanwhile, the expression of miR-152 was negatively correlated with DNMT1 expression. These findings suggest that the DNA hypermethylation-induced downregulation of miR-152 may be related to bladder carcinogenesis. The specific molecular mechanisms between 


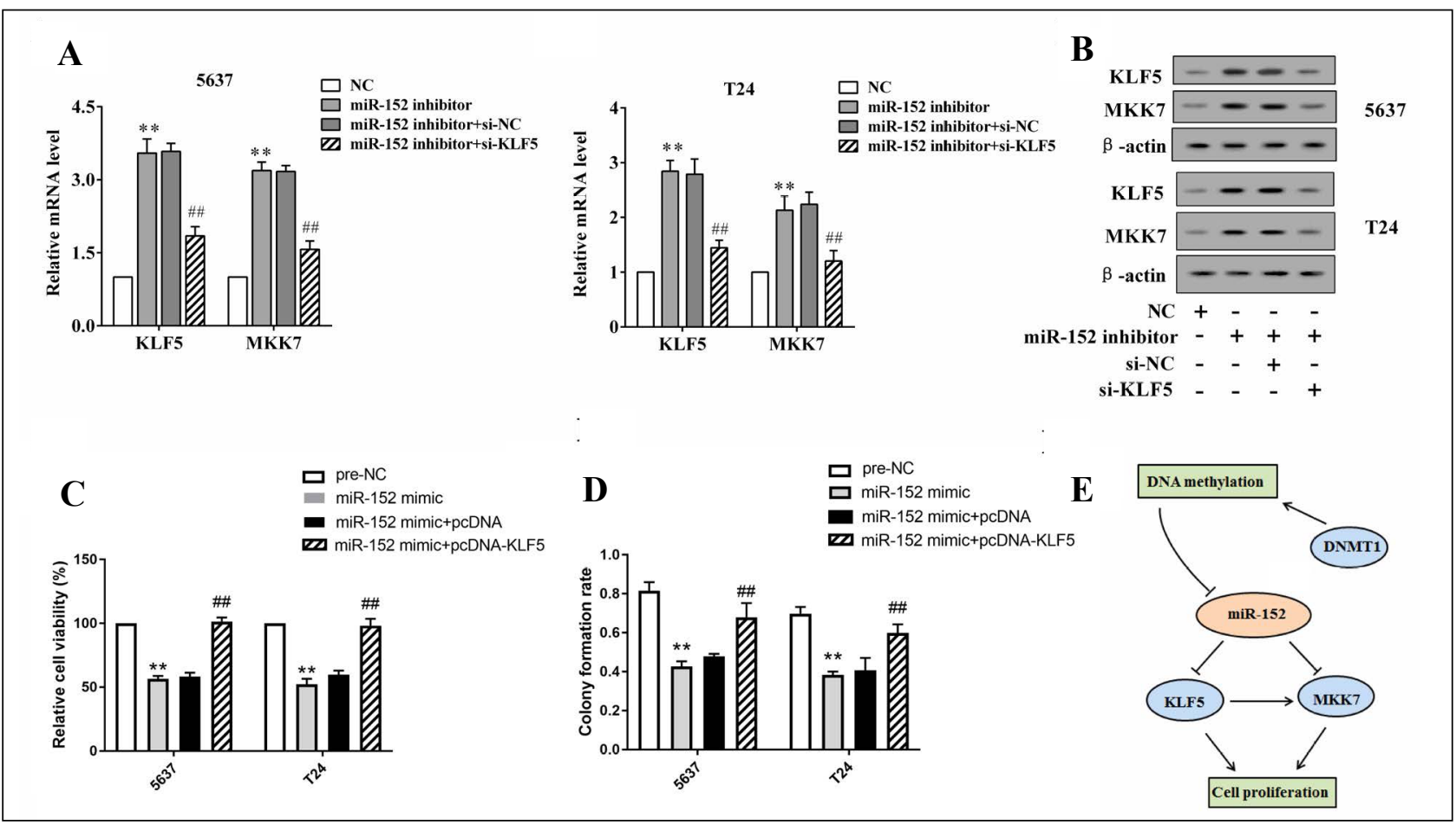

Figure 4. The overexpression of miR-152 suppresses cell proliferation by modulating KLF5 and MKK7. The 5637 and T24 cells were transfected with miR-152 inhibitor or co-transfected with siRNA against KLF5 (si-KLF5). The mRNA levels (A) and the protein levels (B) of KLF5 and MKK7 were detected using qRT-PCR and western blot analysis, respectively. The 5637 and T24 cells were transfected with miR-152 mimic or cotransfected with pcDNA-KLF5. The cell viability (C) and the colony formation rate (D) were detected using an MTT assay and a colony formation assay respectively. (E) A schematic diagram shows that miR-152/KLF5/CKK6 and DNMT1 established a bi-loop to regulate bladder cancer. $* * P<0.01$ vs controls (NC and pre-NC). \#\# $P<0.05$ vs miR-152 inhibitor + si-NC or miR-152 mimic + pcDNA.

miR-152 and DNMT1 merit further investigation.

The silencing of the target gene is the major function of miRNAs. By binding with the 3'UTR of the complementary sequences in mRNAs, miRNAs accumulate RNA-binding proteins like Ago2, and they block gene expression by breaking down the targeted mRNAs [12]. Notably, we searched the online database Targetscan and found binding sites at the 3'UTR of the KLF5 and MKK7 mRNAs that are predicted to be complemented by miR152. The luciferase reporter assay confirmed the target relationship between miR-152 and KLF5, as well as MKK7. Interestingly, the transcriptional factor KLF5 has been demonstrated to positively regulate MKK 7 expression by binding with MKK7 promoter regions [11]. Our findings also indicate that MKK7 transcription was promoted by KLF5. We suppose that the overexpression of miR-152 not only directly suppressed KLF5 and MKK7 expression via 3'UTR binding but also indirectly suppressed KLF5 and MKK 7 expression via the transcriptional inhibition of MKK7. Furthermore, the inhibition of bladder cancer cell proliferation was proven to be modulated by the miR-152/ KLF5/MKK7 axis.

In conclusion, the present study indicated that miR-152 was downregulated in bladder cancer cell lines. This downregulation of miR-152 was partly associated with the DNA hypermethylation mediated by DNMT1. The overexpression of miR-152 suppressed bladder cancer cell proliferation and tumor growth both in vitro and in vivo, which was modulated by targeting KLF5 and MKK7. Our findings provide a novel insight into the mechanism of miR-152 in bladder cancer tumorigenesis.

\section{Declaration}

Conflict of Interest: The authors declare that they have no conflict of interest.

\section{References}

1. Herr H, Konety B, Stein J, et al. Optimizing outcomes at every stage of bladder cancer: Do we practice it?. Urologic Oncology: Seminars and Original Investigations, 2009, 27(1):0-74.

2. Barocas D A, Globe D R , Colayco D C , et al. Surveillance and Treatment of Non-Muscle-Invasive Bladder Cancer in the USA. Advances in Urology, 2012, 2012:1-8.

3. Wu Q, Fu C, Li M, et al. CINP is a novel cofactor of KLF5 required for its role in the promotion of cell proliferation, survival and tumor growth. International journal of cancer, 2019, 144(3): 582-594.

4. Chen C, Benjamin M S, Sun X, et al. KLF5 promotes cell proliferation and tumorigenesis through gene regulationin the TSU-Pr1 human bladder cancer cell line. International Journal of Cancer, 2006, 118(6):1346-1355.

5. KLF5 promotes cell migration by up-regulating FYN in bladder cancer cells. FEBS Letters, 2016, 590(3). 
6. Seger R, Krebs E G. The MAPK signaling cascade. The FASEB journal, 1995, 9(9): 726-735.

7. Zhang W, Liu H T. MAPK signal pathways in the regulation of cell proliferation in mammalian cells. Cell research, 2002, 12(1): 9.

8. Johnson G L, Lapadat R. Mitogen-activated protein kinase pathways mediated by ERK, JNK, and p38 protein kinases. Science, 2002, 298(5600): 1911-1912.

9. Qiu F, Yang L, Lu X, et al. The MKK7 p. Glu116Lys rare variant serves as a predictor for lung cancer risk and prognosis in Chinese. PLoS genetics, 2016, 12(3): e1005955.

10. Sakai H, Sato A, Aihara Y, et al. MKK 7 mediates miR-493dependent suppression of liver metastasis of colon cancer cells. Cancer science, 2014, 105(4): 425-430.

11. Wang Y, Xia Y, Hu K, et al. MKK7 transcription positively or negatively regulated by SP1 and KLF5 depends on HDAC4 activity in glioma. International journal of cancer, 2019.

12. Beermann J, Piccoli M T, Viereck J, et al. Non-coding RNAs in development and disease: background, mechanisms, and therapeutic approaches. Physiological reviews, 2016, 96(4): 1297-1325.

13. Kaikkonen M U, Adelman K. Emerging roles of non-coding RNA transcription. Trends in biochemical sciences, 2018, 43(9): 654-667.

14. Maimaitiming A, Wusiman A, Aimudula A, et al. microRNA-152 inhibits cell proliferation, migration and invasion in breast cancer. Oncology Research Featuring Preclinical and Clinical Cancer Therapeutics, 2019.

15. Feng F, Liu H, Chen A, et al. miR-148-3p and miR-152-3p synergistically regulate prostate cancer progression via repressing KLF4. Journal of cellular biochemistry, 2019.

16. Köhler C U, Bryk O, Meier S, et al. Analyses in human urothelial cells identify methylation of miR-152, miR$200 \mathrm{~b}$ and miR-10a genes as candidate bladder cancer biomarkers. Biochemical and biophysical research communications, 2013, 438(1): 48-53.

17. Jiang X, Du L, Wang L, et al. Serum microRNA expression signatures identified from genome-wide microRNA profiling serve as novel noninvasive biomarkers for diagnosis and recurrence of bladder cancer. International journal of cancer, 2015, 136(4): 854-862.
18. Wang H, Zhang W, Zuo Y, et al. miR-9 promotes cell proliferation and inhibits apoptosis by targeting LASS2 in bladder cancer. Tumor Biology, 2015, 36(12): 96319640.

19. Xie D, Shang C, Zhang H, et al. Up-regulation of miR9 target CBX7 to regulate invasion ability of bladder transitional cell carcinoma. Medical science monitor: international medical journal of experimental and clinical research, 2015, 21: 225.

20. Zhang H H, Qi F, Cao Y H, et al. Expression and clinical significance of microRNA-21, maspin and vascular endothelial growth factor-C in bladder cancer. Oncology letters, 2015, 10(4): 2610-2616.

21. Feng Y, Liu J, Kang Y, et al. miR-19a acts as an oncogenic microRNA and is up-regulated in bladder cancer. Journal of experimental \& clinical cancer research, 2014, 33(1): 67.

22. Miah S, Dudziec E, Drayton R M, et al. An evaluation of urinary microRNA reveals a high sensitivity for bladder cancer. British journal of cancer, 2012, 107(1): 123.

23. Pençe S, Özbek E, Tiryakioğlu N O, et al. Deregulation of seven $\mathrm{CpG}$ island-harboring miRNAs in bladder cancer: miR-155 and miR-23b as the most promising oncomiRs. Cellular and molecular biology, 2016, 62(3): 25-30.

24. Majid S, Dar A A, Saini S, et al. MicroRNA-23b functions as a tumor suppressor by regulating Zeb1 in bladder cancer. PloS one, 2013, 8(7): e67686.

25. Zhang D Z, Lau K M, Chan E S Y, et al. Cell-free urinary microRNA-99a and microRNA-125b are diagnostic markers for the non-invasive screening of bladder cancer. PloS one, 2014, 9(7): e100793.

26. Du M, Shi D, Yuan L, et al. Circulating miR-497 and miR$663 \mathrm{~b}$ in plasma are potential novel biomarkers for bladder cancer. Scientific reports, 2015, 5: 10437.

27. Richardson B C, Patel D R. Epigenetics in 2013: DNA methylation and miRNA-key roles in systemic autoimmunity. Nature Reviews Rheumatology, 2014, 10(2): 72.

28. Keating S T, El-Osta A. Epigenetics and metabolism. Circulation research, 2015, 116(4): 715-736.

29. Kinnaird A, Zhao S, Wellen K E, et al. Metabolic control of epigenetics in cancer. Nature Reviews Cancer, 2016, 16(11): 694-707.

Cite this article as: Hui Y, Huang Y, Ding X, et al. MicroRNA-152 suppresses cell proliferation and tumor growth of bladder cancer by targeting KLF5 and MKK7 [J]. Aging Pathobiology and Therapeutics, 2019, 1(1): 10-16. 